\title{
Morphology of the Venus Ultraviolet Night Airglow
}

\author{
A. I. F. Stewart, J.-C. Gérard, ${ }^{1}$ D. W. Rusch, AND S. W. Bougher \\ Laboratory for Atmospheric and Space Physics and Department of Astrogeophysics \\ University of Colorado, Boulder, Colorado 80309
}

\begin{abstract}
Images of the nightside of Venus in the $(0,1) \delta$ band of nitric oxide have been obtained by the Pioneer Venus orbiter ultraviolet spectrometer (OUVS). The emission, which is produced by radiative association of $\mathrm{N}$ and $\mathrm{O}$, shows a bright spot reaching $\sim 5 \mathrm{kR}$ and located at $2 \mathrm{a} . \mathrm{m}$. local solar time just south of the equator. The emitting layer is at $111 \pm 7 \cdot \mathrm{km}$ altitude. A one dimensional vertical transport model shows that the hemispheric average brightness of $0.8 \mathrm{kR}$ is consistent with the orbiter neutral mass spectrometer (ONMS) measurements of $N$ and $O$ near $167 \mathrm{~km}$, and that the altitude of the emitting layer is consistent with the eddy mixing model proposed to explain the dayside helium profile measured by the bus neutral mass spectrometer. In the model, $N$ reaches a peak of $7 \times 10^{8} \mathrm{~cm}^{-3}$ at $114 \mathrm{~km}$, and $\mathrm{O}$ reaches a peak of $2.6 \times 10^{11} \mathrm{~cm}^{-3}$ at $106 \mathrm{~km}$. There is a fair degree of consistency between the ONMS, OUVS, and other airglow measurements, except as regards the local time dependence.
\end{abstract}

\section{INTRODUCTION}

The ultraviolet night airglow of Venus was first detected by Barth et al. [1968] from Mariner 5. Subsequently, the visual Herzberg II and Chamberlain bands of molecular oxygen were seen from Venera 9 and 10 [Krasnopolsky et al., 1976; Lawrence et al., 1977; Slanger and Black, 1978]. The ultraviolet nightglow was again detected from Pioneer Venus [Stewart et al., 1979] and subsequently identified as the gamma and delta bands of nitric oxide [Feldman et al., 1979; Stewart and Barth, 1979] excited by the radiative association of atoms of nitrogen and oxygen. Since in the spectrum of the night airglow the emissions that might be expected to accompany the dissociative production of $\mathrm{O}$ and $\mathrm{N}$ are weak or absent [Stewart and Barth, 1979; Stewart, 1980], it is evident that the night airglow is a result of the transport of atoms from the dayside by the general circulation of the thermosphere.

Stewart and Barth [1979] reported that the maximum emission apparently occurred near the equator at about 2 a.m. local solar time and that bright patches were occasionally seen elsewhere. Since then, the precession of the orbit of the Pioneer Venus spacecraft through local time has allowed the ultraviolet spectrometer to obtain numerous images of the dark side of Venus showing the spatial and temporal variation of the emission. In the present paper we describe these images and their analysis. We also determine the altitude of the emitting layer, using data from an earlier phase of the mission when periapsis was on the nightside.

We investigate the production of the emission using a simple one dimensional steady state model of the transport of $\mathrm{N}$ and $\mathrm{O}$ downward through the nightside thermosphere. The comparison of this model with our observations confirms the plausibility of the concept of the dayside origin of the atoms and confirms also that vertical mixing on the nightside is very vigorous.

\section{OBSERVATIONS}

The orbit of the Pioneer Venus spacecraft has been described elsewhere [Colin, 1979a, b], as has the geometry of the

\footnotetext{
' On leave from Institut d'Astrophysique, Université de Liège, Liège, Belgium.

Copyright $\odot 1980$ by the American Geophysical Union.
}

ultraviolet spectrometer observations of the planet [Stewart, 1980]. The essential characteristics are presented in Figure 1. The slant range from the spacecraft to the limb seen by the ultraviolet spectrometer varies from $31900 \mathrm{~km}$ at $P-2: 03(2 \mathrm{~h}$, $3 \mathrm{~min}$ before periapsis) to $11700 \mathrm{~km}$ at $P-0: 33$, and from $6100 \mathrm{~km}$ at $P-0: 16$ to $<1000 \mathrm{~km}$ at periapsis to $4100 \mathrm{~km}$ at $P$ $+0: 10$. The planet is in view for a maximum of $36^{\circ}$ of spin during the first view period and for a maximum of $186^{\circ}$ during the second.

The images that concern us here were obtained from orbit 167 (May 20, 1979) through orbit 210 (July 2, 1979), during which period the subspacecraft point at $30^{\circ} \mathrm{N}(\sim P-0: 55)$ moved from 2225 to 0300 local time. The images were acquired in a series of swaths, normally $36 \mathrm{~s}$ but occasionally $24 \mathrm{~s}$ apart. The integration period used was $8 \mathrm{~ms}$, giving an angular resolution across the planet of $0.23^{\circ}$. Resolution in the north-south direction was determined by the length of the slit, $1.38^{\circ}$; there was considerable overlap of successive swaths. The wavelengths used were $1980 \AA$ (the 0,1 delta band) and $2365 \AA$ (the 0,1 gamma band). We concentrate on the $1980-\AA$ images, which are less subject to contamination by internally scattered light near the terminators than are the 2365- $\AA$ images.

Images obtained during the second view period were obtained with a $32-\mathrm{ms}$ integration period ( $0.94^{\circ}$ of spin). Because of the lower spacecraft altitude, successive swaths do not overlap, and in addition, the changes in range are much more severe. Several such images were analyzed by Stewart and Barth [1979]; none are considered here. However, swaths from these images taken near periapsis were used to determine the altitude of the emitting layer. Near periapsis the long axis of the slit aligns with the limb and this, combined with the low altitude and short slant ranges, gives good altitude resolution on the limb. Although $\$ 5-\mathbf{k m}$ resolution is possible using a $4 \mathrm{~ms}$ integration period, small uncertainties in the data timing prevent an absolute altitude determination at this resolution. The method used was to select swaths that clearly show both limbs in the limb-brightened airglow emission, and to resolve the uncertainties in timing by assuming that the emission layer altitude is the same on both limbs. The disadvantage here is the $32-\mathrm{ms}$ integration period, which degrades the altitude resolution to $\sim 20 \mathrm{~km}$. The remaining uncertainty in the emission altitude arises from the small 


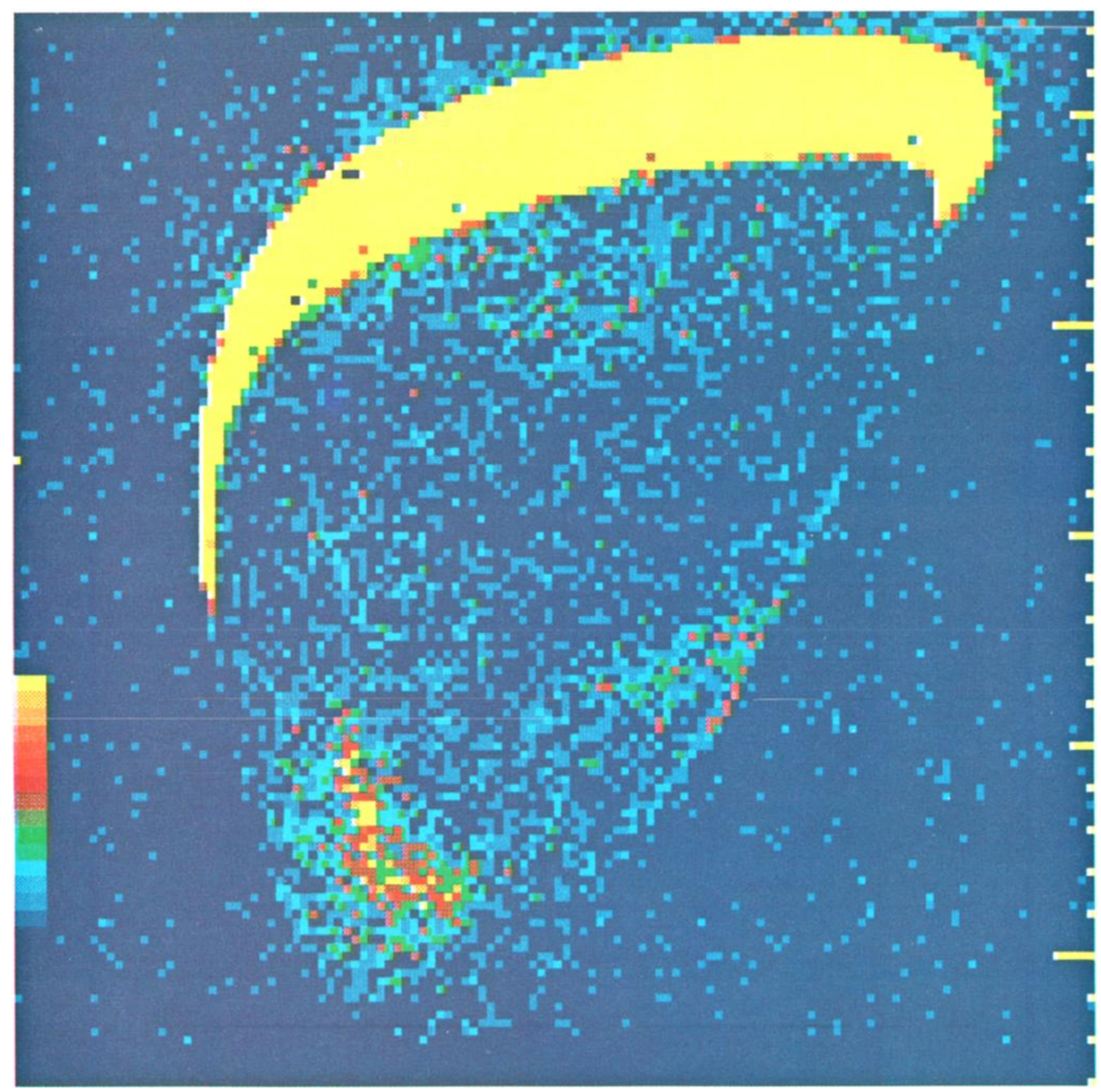

Plate 1. Unrectified image at $1980 \AA$ obtained on orbit 186 . The color code is shown at the lower left and the scale is 1 $\mathrm{kR}$ per color level. The sunlit crescent is set to $15 \mathrm{kR}$. The blue spots outside the planet disc are due to instrument background. 


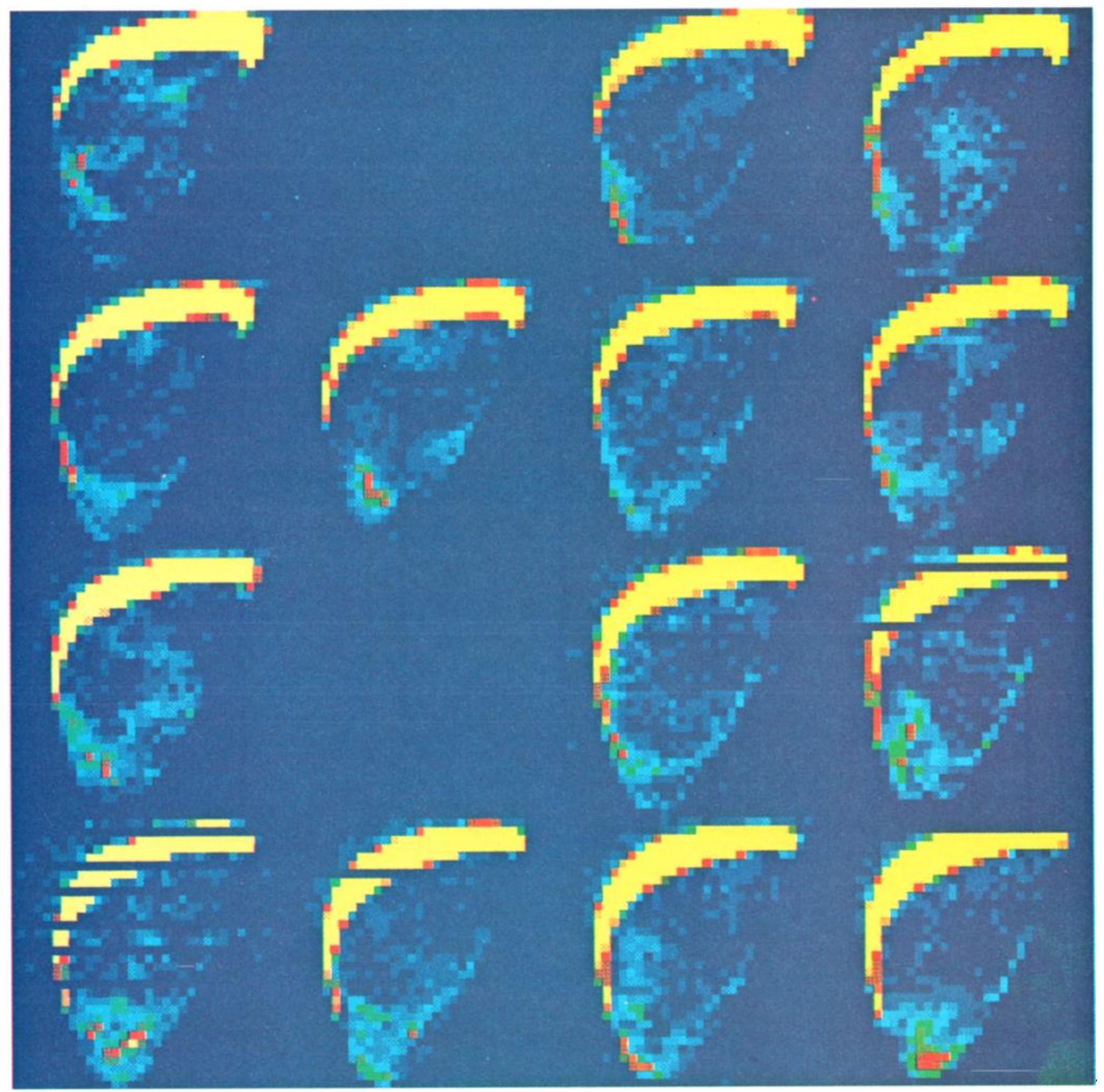

Plate 2. Consecutive unrectified images observed between orbits 181 and 196 . The empty cases correspond to orbits when no NO $\delta$ or $\gamma$ observations were available. The color scale is the same as in Plate 1. 


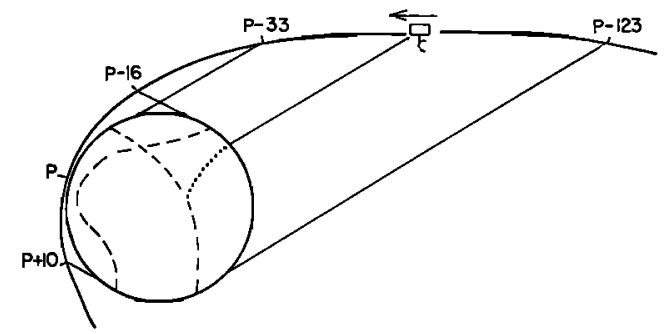

Fig. 1. Drawing (not to scale) of the OUVS observing geometry. The dotted line represents part of a typical trace across the disc (the remainder is on the other side of the planet). The dashed lines represent the envelopes of the endpoints of these traces, i.e., the limb of the planet as seen by OUVS.

changes in spin period and in spin axis orientation that occur near periapsis due to aerodynamic drag and to the deployment of the radar antenna.

The presence of the diffusely reflecting cloud layer beneath the airglow layer enhances the observed signal at wavelengths $>2000 \AA$, but at $1980 \AA$ the single-scattering albedo of $\mathrm{CO}_{2}$ is 0.25 , and the enhancement is less than $10 \%$. The instrument sensitivity at $1980 \AA$ is $1.5 \mathrm{c} / \mathrm{kR} / 32 \mathrm{~ms}$.

\section{RESULTS}

Apoapsis images obtained between May 20 and July 2, 1979 , in the wavelength of the $(1,0) \delta$ band have been analyzed to study the Venus nitric oxide nightglow emission. Plate 1 shows an example of a false color unrectified image at $1980 \AA$ obtained during orbit 186 on June 8,1979 . The intensity scale has 15 levels ranging from 1 (dark blue) to 15 (bright yellow) $k R$. The color scale is displayed at the lower left of the image. In this particular representation, intensities equal to or larger than $15 \mathrm{kR}$ are set to the 15 th color level. The yellow crescent near the top of the image corresponds to the sunlit portion of the disc where the intensity of the scattering of solar radiation on the dayside greatly exceeds the 15-kR limit. The coordinates of the image are spin phase angle in abscissa and time from periapsis in ordinate. The tick marks along the vertical axis correspond to 4-min time intervals from periapsis from $P$ -126 to $P-32 \mathrm{~min}$. Some averaging of adjacent data points has been performed in order to present the image in a $128 \times$ 128 pixel format. The distorted aspect of the planet's disc is caused by the motion of the spacecraft along its inclined orbit during the $\sim 90$ min necessary to scan the planet.

The disc of the planet is readily distinguished from the background where the count rate is substantially smaller than on the disc, indicating the presence of the nightside airglow. The airglow distribution is structured with emission rates ranging from less than 1 to $15 \mathrm{kR}$ in this particular image. A bright area is clearly visible in the southern hemisphere, and the attitude data indicates that it is located near the equator in the morning sector.

Since one apoapsis image of the planet is obtained every 24 hours, the opportunity is given to examine the variability of the airglow structure. A set of 14 images at $1980 \AA$ from orbit 181 to 196 is displayed in Plate 2 in chronological order. The missing images correspond to orbits 182 and 190 when Ly $\alpha$ observations were made. In order to insert the images into a $128 \times 128$ array, each individual image was compressed linearly by a factor of 4 . Consequently, 16 elements of the unrectified images are averaged to make one element of Plate 2.
The color scale is the same as in Plate 1. The displacement of the terminator with respect to the limb is due to the revolution of the planet about the sun by $1.60 \% / 24$ hours. Occasional dark stripes across the disc are due to a loss of telemetry data or to scans made in other wavelengths than the NO $\delta(0,1)$ band. Large variations of the airglow are readily seen both in intensity and morphology. From these and other images, we find no clear periodicity in either the average or the peak brightness (cf. Figure 2). The morning sector bright spot is usually present, and the equator is generally brighter than other latitudes. Random bright patches are seen to occur at all latitudes and local times, with the exception of the mid-latitude region near the evening terminator (in the upper right of these images).

Maps in ecliptic latitude and longitude coordinates have been made for orbits 167 to 210 , following the procedure outlined below. Each individual monochromatic image is first corrected for the emission angle. The angle from nadir is calculated for each image element, and the corresponding intensity is converted into a vertical emission rate by applying the appropriate Chapman function; the topside scale height of the airglow distribution is taken to be $5 \mathrm{~km}$, and the peak of the layer is assumed to be located at $110 \mathrm{~km}$. The image elements are subsequently sorted out into latitude and longitude bins of $3^{\circ} \times 4.8^{\circ}$ size, respectively. Ecliptic longitudes are finally converted into hour angle by shifting the longitude scale by an amount depending on the date of observation. The map obtained on orbit 186 is shown in Plate 3, superimposed on a latitude and hour angle grid. In this case the color scale is 400 $R$ per color level from 0 to $6 \mathrm{kR}$, and as before, intensities exceeding the maximum value have been set to color level 15 . Because of the viewing geometry the grid cannot be entirely filled in since the most southern latitude seen by the OUVS instrument is $60^{\circ}$ and other parts of the planet are not reached by the field of view. The high intensity levels along the $90^{\circ} \mathrm{N}$ latitude line and the $90^{\circ}$ and $270^{\circ}$ hour angle meridans are caused by contamination of the observations by scattered light originating from the dayside. The center of the bright airglow spot seen in Plate 1 is located near $15^{\circ} \mathrm{S}$ latitude and $0130 \mathrm{LT}$. The fainter bright area near the limb in Plate 1 is also visible on the map, but the maximum emission rate is no longer situated on the limb as a consequence of the correction applied for the emission angle.

In order to investigate the statistical morphology of the NO $\delta$ nightglow, a latitude hour angle map was constructed for each of 35 available images between orbits 167 and 210 . The dayside and the scattered light contributions were removed from each map, and the resulting nightside maps were added together. The intensity was then normalized by the total number of entries contributing to each bin of the final map. This global map is shown in Plate 4 on a $200 \mathrm{R}$ per color level scale. As was expected from the individual maps, it shows a bright patch reaching a maximum brightness of $7.5 \mathrm{kR}$ and centered near $10^{\circ} \mathrm{S}$ and $0200 \mathrm{LT}$. In addition, the equatorial region in the premidnight and a high-latitude area in the postmidnight sector also exhibit secondary intensity maxima. A relatively dark region near $40^{\circ} \mathrm{N}$ and $2000 \mathrm{LT}$ is also seen. The areas located north of $75^{\circ} \mathrm{N}$ or having hour angles greater than $60^{\circ}$ and less than $285^{\circ}$ have been excluded by the removal of the daylight. The average vertical emission rate deduced from this map is $780 \mathrm{R}$ for the NO $\delta(0,1)$ band, which corresponds to $2.7 \mathrm{kR}$ for the total NO $\delta$ system. 
Since large day-to-day variations are observed in the airglow morphology, a systematic study of its variability was made, using the set of orbits included in Plate 4 . The average intensity in a rectangle bounded by $60^{\circ} \mathrm{S}$ and $66^{\circ} \mathrm{N}$ and by $2000 \mathrm{LT}$ and $0400 \mathrm{LT}$ was calculated. The result is shown in Figure 2 by the curve labelled 'dark disk average,' where the dots represent the average emission rate as a function of the orbit number. The intensity variation of the brightest area of the nightside is also plotted at the top of Figure 2 on the same intensity scale. The emission rate is obtained by averaging the intensity over a $9^{\circ}$ of latitude $\times 15^{\circ}$ of hour angle area centered on the brightest image element. Finally, the $F 10.7 \mathrm{~cm}$ solar flux is plotted at the bottom. The fluxes are measured from the earth and shifted in date to account for the fact that the two planets are at different solar longitude. This delay is given in days by $\Delta T=27 \alpha / 360$, where $\alpha$ is the difference in longitude between the two planets.

The curves show fluctuations by almost a factor of 2 of the average brightness. The variations of the maximum brightness are even larger but generally in phase with the total nightside intensity. These fluctuations are statistically significant due to the large number of counts included in each map. Each raw image of the nightside contains $\sim 10^{4}$ counts, and consequently, the standard deviation for each point of the average brightness curve is on the order of $1 \%$, much smaller than the measured day-to-day variations. The standard deviation for the points of the maximum brightness curve is on the order of $5 \%$. There is no evidence of correlation between the two brightness curves and the solar flux, and in fact, the average brightness keeps increasing after orbit 198 when the solar activity decreases. This trend is caused by the change in local time of the orbital plane since the early images tend to emphasize the evening sector, whereas the later ones mostly include the morningside. The intensity of the brightest spot remains almost constant since the bright area near 2 a.m. is within the area covered by all the images used in Figure 2.

\section{INTERPRETATION}

We have used a one dimensional chemical diffusion model similar to that employed by Rusch and Cravens [1979] to solve for the density distributions of $\mathrm{CO}, \mathrm{N}, \mathrm{O}, \mathrm{O}_{2}, \mathrm{NO}$, and $\mathrm{O}_{3}$ on the nightside of Venus. The upper and lower boundaries in the model are at $140 \mathrm{~km}$ and $70 \mathrm{~km}$, respectively. Table $1 \mathrm{de}-$ tails the chemical reactions employed and the adopted rate coefficients. We compare the results of the model with the OUVS airglow measurements of the emission in the nitric oxide delta bands formed by reaction 1 .

The downward flowing atomic nitrogen is consumed by radiative association with atomic oxygen, with a rate coefficient $k$; the atomic oxygen subsequently recombines at a lower altitude. In this situation the $\mathbf{N}$ and $\mathbf{O}$ satisfy some simple relationships, which we demonstrate by using a one dimensional analytic model. For simplicity, we assume that the thermosphere is isothermal and that eddy transport greatly exceeds transport by molecular diffusion. Then, in the absence of sources and sinks, the vertical flux $\phi$ and number density $n$ of a minor constituent satisfy the equation

$$
\begin{gathered}
\phi=-K\left(\frac{d n}{d z}+\frac{n}{H}\right) \\
\frac{d \phi}{d z}=0
\end{gathered}
$$

where $K$ is the eddy coefficient and $H$ is the ambient scale height. If we adopt the form $K=A / M^{1 / 2}$ [von Zahn et al., this issue], where $M$ is the ambient number density and $A$ is a constant, then the solution to these equations is

$$
n=-\frac{2 \phi H}{K}+B \cdot M
$$

where $B$ is a constant of integration (this solution can be verified by substitution). For a minor constituent flowing downward from a high-altitude source to sink at lower altitudes, the appropriate value for $B$ is zero. Thus for atomic oxygen introduced by high-altitude horizontal transport from the dayside, we have at altitudes above its recombination sink

$$
[O]=2\left|\phi_{\mathrm{o}}\right| H / K
$$

where $[O]$ and $\phi_{0}$ are the number density and vertical flux of atomic oxygen, respectively. We assume that this expression describes the oxygen density at the altitudes where $N$ is being consumed by radiative association with $O$. A good rule of thumb in problems of downward transport into a chemical sink region is that the minor constituent reaches its maximum density at the altitude where the transport and chemical lifetimes are equal [Rishbeth and Barron, 1960]; that is, for atomic nitrogen, where

$$
H^{2} / K=(k[O])^{-1}
$$

Using our expressions for [O] and $K$ it is readily seen that at this altitude,

$$
\begin{gathered}
M=M_{p}=A^{2} / 2 k\left|\phi_{\mathrm{O}}\right| H^{3} \\
{[\mathrm{O}]=[\mathrm{O}]_{p}=\left(2\left|\phi_{\mathrm{O}}\right| / k H\right)^{1 / 2}}
\end{gathered}
$$

and the chemical lifetime of $\mathrm{N}$ is

$$
\left(\tau_{\mathrm{N}}\right)_{p}=\left(k[\mathrm{O}]_{p}\right)^{-1}=\left(H / 2 k\left|\phi_{\mathrm{O}}\right|\right)^{1 / 2}
$$

Thus the altitude of the maximum $\mathrm{N}$ density depends on $A$ (i.e., on the strength of eddy mixing) and on the downward flux of $O$, while the chemical lifetime of $N$ at its peak depends on the flux of $O$ but not on the eddy mixing coefficient. For example, for $A=2 \times 10^{13}$ [von Zahn et al., 1979] and $\left|\phi_{\mathrm{O}}\right|=1$ $\times 10^{12} \mathrm{~cm}^{-2} \mathrm{~s}^{-1}$, we find $M_{p}=1.5 \times 10^{14} \mathrm{~cm}^{-3}$, corresponding to about $110 \mathrm{~km}$; at this altitude, $\tau_{N}=8 \times 10^{4} \mathrm{~s}$ or about 1 day.

We have performed a detailed vertical transport calculation to extend the above arguments. We chose a vertical (downward) flux of $1 \times 10^{12} \mathrm{~cm}^{-2} \mathrm{~s}^{-1}$ for $O$ and $1 \times 10^{10} \mathrm{~cm}^{-2} \mathrm{~s}^{-1}$ for N. The value for $\phi_{o}$ is representative of values calculated by Dickinson and Ridley [1977], while the value for $\mathrm{N}$ is simply scaled from this by the ratio of the $\mathrm{N}$ and $\mathrm{O}$ densities [Niemann et al., this issue]. The results of the calculation are shown in Figure 3 for a value of $K_{140}=3 \times 10^{8} \mathrm{~cm}^{2} \mathrm{~s}^{-1}$ (i.e., $A=1.6$ $\times 10^{13}$ ). The atomic oxygen and nitrogen measurements of Niemann et al. [this issue] extrapolated to $140 \mathrm{~km}$ assuming diffusive equilibrium agree with our values there to within a factor of 2 . The atomic oxygen profile has a maximum density at $106 \mathrm{~km}$ of $2.6 \times 10^{11} \mathrm{~cm}^{-3}$, and the maximum nitrogen density is $7.3 \times 10^{8} \mathrm{~cm}^{-3}$ at $114 \mathrm{~km}$. The atomic oxygen density at the nitrogen peak is $2 \times 10^{11} \mathrm{~cm}^{-3}$ in reasonable agreement with the value $3 \times 10^{11} \mathrm{~cm}^{-3}$ predicted by our simple theoretical treatment. The curve labeled $P\left(\mathrm{O}_{2}\right)$ is the production rate of molecular oxygen by reaction 4 of Table 1 . We will discuss its importance later in this section. 


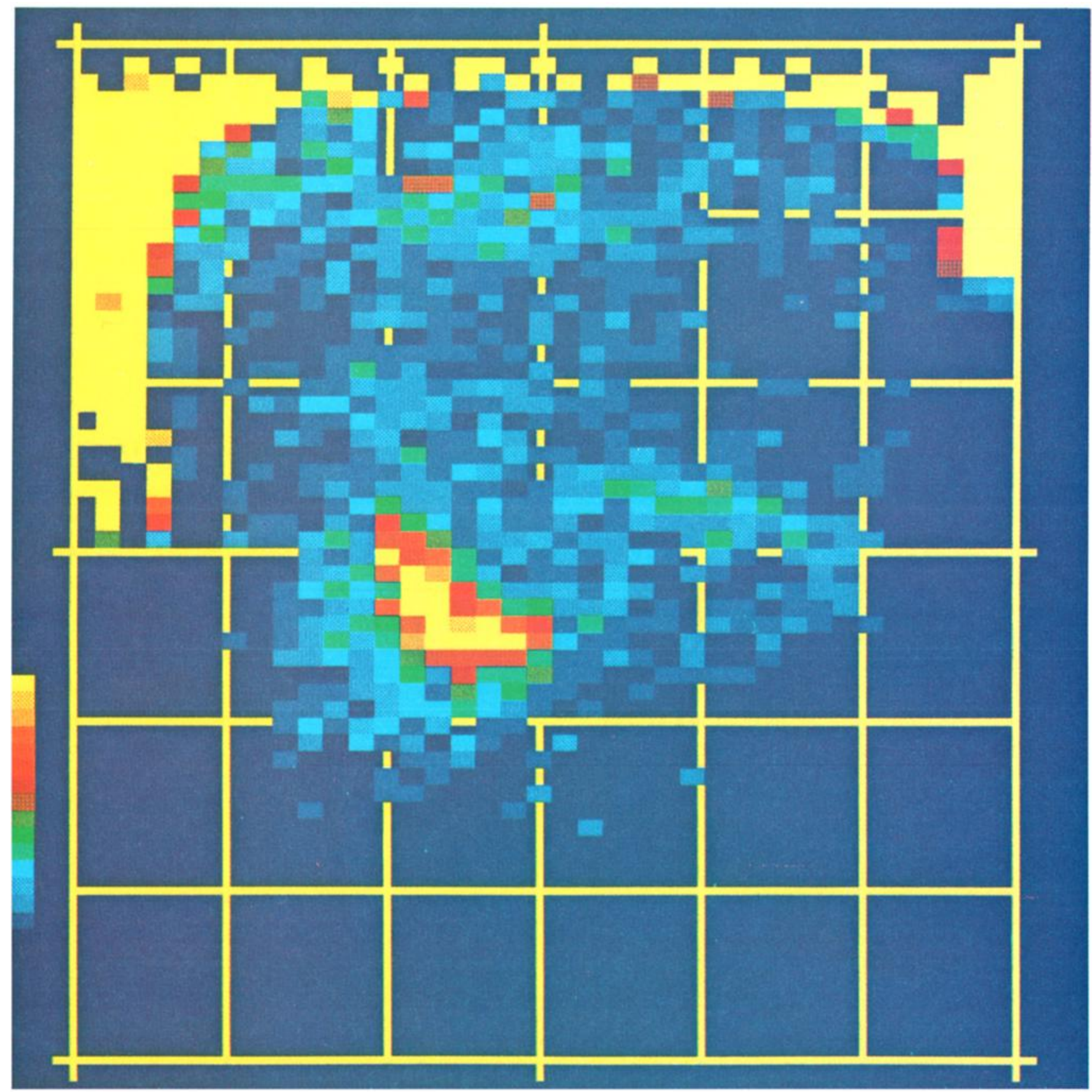

Plate 3. Mercator projection of the image from orbit 186 (Plate 1). Lines are plotted every $30^{\circ}$ of latitude from $90^{\circ} \mathrm{N}$ to $90^{\circ} \mathrm{S}$ and hour angle from $270^{\circ}$ (right) to $90^{\circ}$ (left). The color scale is $400 \mathrm{R}$ per color level and the yellow regions are contaminated by instrumentally scattered light. 


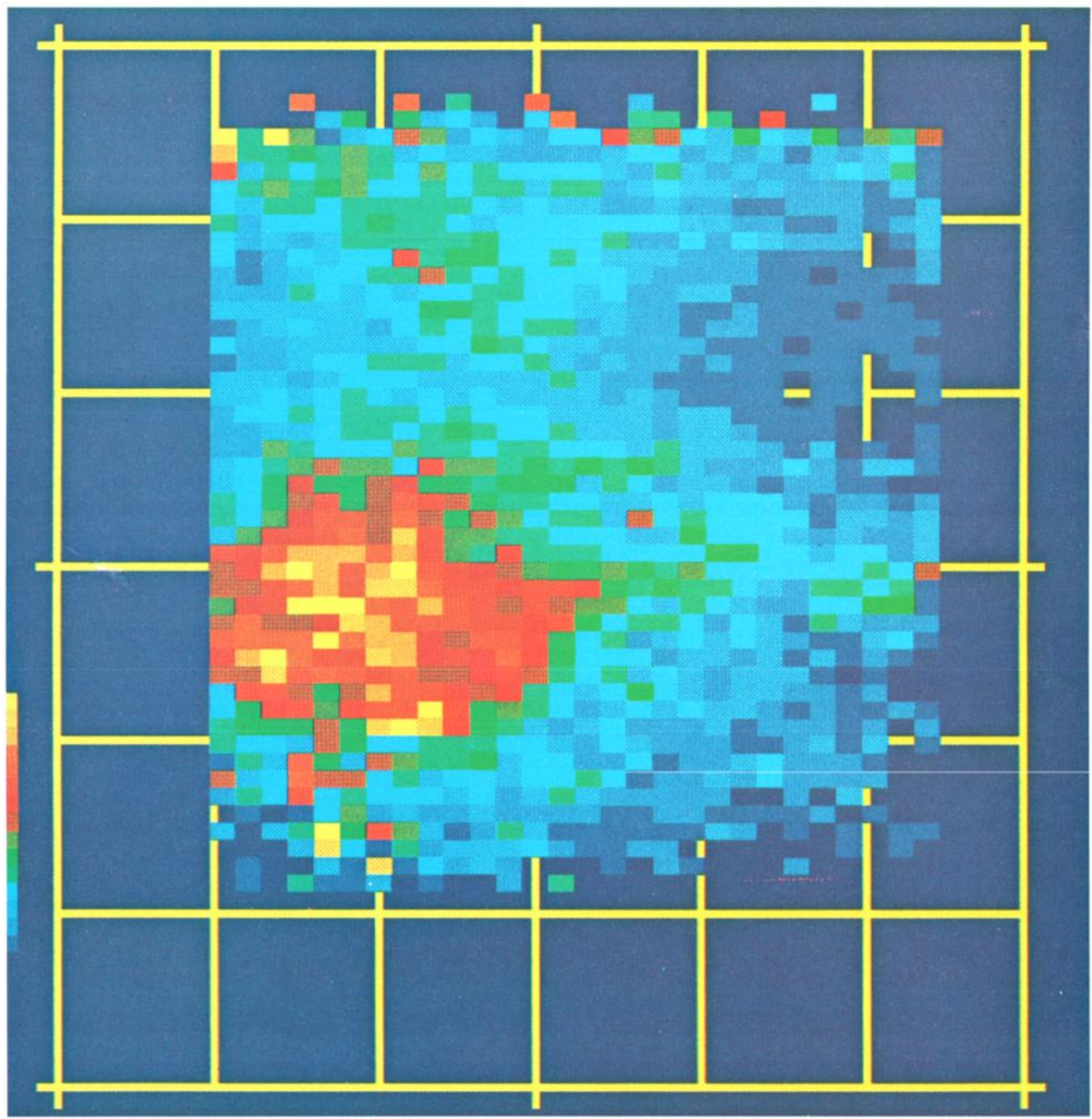

Plate 4. Map of the NO $\delta(0,1)$ band vertical intensity distribution obtained by summing up 35 orbits. The latitude and hour angle grid are similar to Plate 3 . The color scale is $200 \mathrm{R}$ per color level. 
PV OUVS: Nitric Oxide $8(0,1)$ Band, $1980 \AA$

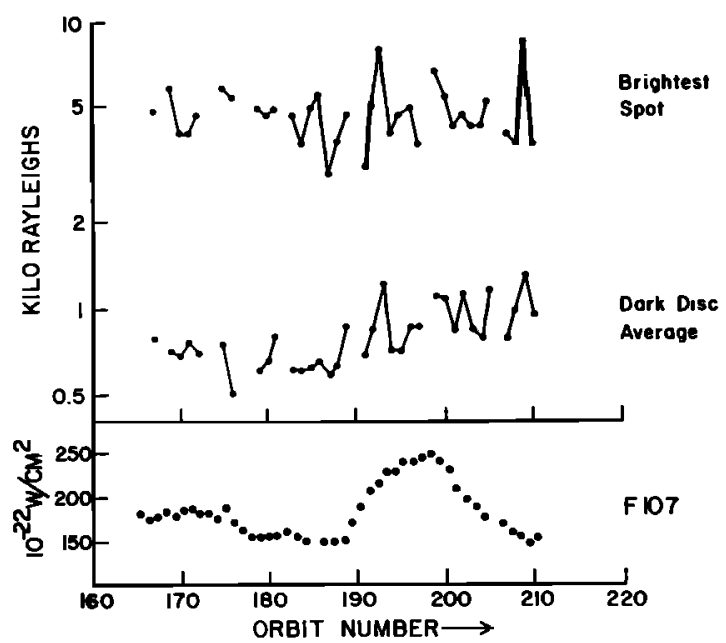

Fig. 2. Variation of the average (lower curve) and maximum (upper curve) brightness in the $(0,1) \delta$ band of NO over 35 orbits. The lower curve is averaged over all latitudes between $60^{\circ} \mathrm{S}$ and $66^{\circ} \mathrm{N}$ and all local times between $8 \mathrm{p} . \mathrm{m}$. and $4 \mathrm{a} . \mathrm{m}$. The upper curve is averaged over a $9^{\circ}$ (latitude) $\times 15^{\circ}$ (hour angle) box containing the brightest spot.

The volume emission rate profile in the nitric oxide delta bands peaks at $110 \mathrm{~km}$ with an intensity of $3.6 \times 10^{3} \mathrm{ph} \mathrm{cm}^{-3}$ $\mathrm{s}^{-1}$. The integrated vertical intensity is $2.8 \mathrm{kR}$, which is in good agreement with the measured average intensity on the nightside of $2.6 \mathrm{kR}$. In the model the efficiency of nitrogen atoms in producing excited NO molecules is determined by the fraction that are lost through reaction 1.

Nitrogen atoms are also lost by reactions 2 and 3 . Reaction 3 is a potential source of gamma and delta emission, but the efficiency for production of an excited NO molecule is extremely small and we ignore its contribution. In an equilibrium calculation, half of the $\mathrm{N}$ atoms must be lost through reaction 2 , the remainder by reactions 1 and 3 . Reaction 1 accounts for $45 \%$ of the loss, and reaction 3 accounts for $5 \%$ of the loss in this model. Thus a photon is produced by $45 \%$ of $\mathrm{N}$ atoms subsiding on the nightside. This efficiency is independent of the $\mathrm{N}$ flux but is dependent on the strength of the vertical mixing. An increase in $K$ results in a deeper penetration of $\mathrm{N}$ atoms into the atmosphere and a subsequent increase in the importance of the three body reaction, reaction 3 relative to reaction 1. Additional calculations show the efficiency of $\mathbf{N}$ atoms in producing photon changes from $\sim 50 \%$ for $K_{140}=1$ $\times 10^{7} \mathrm{~cm}^{2} \mathrm{~s}^{-1}$ to $36 \%$ for $K_{140}=1 \times 10^{9} \mathrm{~cm}^{2} \mathrm{~s}^{-1}$.

The strength of the vertical mixing affects the altitude at which the atomic oxygen and nitrogen density profiles maxi- mize and the altitude of the maximum volume emission rate. This dependence is shown in Figure 4. The curve labeled $P\left(\mathrm{O}_{2}\right)$ represents the altitude where the production of $\mathrm{O}_{2}$ from reaction 4 is maximum. The nitrogen density and nitric oxide emission rate maxima are more sensitive to $K$ than the atomic oxygen and $\mathrm{O}_{2}$ production rate maxima. For the values of $K$ used the maximum density of $\mathrm{N}$ changes from $133 \mathrm{~km}$ to 107 $\mathrm{km}$, a difference of $26 \mathrm{~km}$, while the $\mathrm{O}$ density maximum changes from 117 to $102 \mathrm{~km}$, a $15 \mathrm{~km}$ difference. Also the curve for the delta band maximum lies parallel to that for the nitrogen density. Thus a determination of the altitude at which the nitric oxide emission rate maximizes is a fairly sensitive indicator of the eddy diffusion coefficient. We have determined the altitude of the emission layer near periapsis on 8 orbits. The results are shown in Figure 5; the mean value is $111 \mathrm{~km}$ with a standard deviation of $7 \mathrm{~km}$. We use only the values obtained before periapsis, since the pulse of aerodynamic drag at periapsis perturbs both the spin rate and the spin axis orientation and renders the pointing information suspect after periapsis. Using Figure 4, this mean altitude implies $K_{140} \sim 3 \times 10^{8} \mathrm{~cm}^{2} \mathrm{~s}^{-1},\left(A \sim 1.6 \times 10^{13}\right)$; this value is consistent with the value $A=2 \times 10^{13}$ derived by von Zahn et al. [1979] near the morning terminator.

\section{DISCUSSION}

Our observations have shown that the nitric oxide night airglow typically shows a bright spot at about 2 a.m., that this spot is $\sim 5$ times brighter than the hemispheric mean brightness, and that the emitting layer is near $111 \mathrm{~km}$. Our one dimensional steady state transport model shows that the zenith emission rate in the NO $\delta$ system is $\sim 0.25$ times the downward flux of $\mathrm{N}$ atoms, that the column production rate of $\mathrm{O}_{2}$ is 0.5 times the downward flux of $\mathrm{O}$ (as expected), and that the altitude of the emitting NO layer depends on the flux of $O$ and on the strength of eddy mixing. A first-order interpretation of our measurements would be that the brightness map is also a map of the downward flux of $N$. Since the $N$ and $O$ have similar atomic weights, we might expect their velocity fields in the thermospheric circulation pattern to be similar, so that the $N$ / $O$ ratio would be fairly uniform over the night hemisphere, at least at altitudes above the chemical sinks.

Several other data sets bear on this question. First, the ONMS in situ measurements of $\mathrm{N}$ and $\mathrm{O}$ [Niemann et al., this issue] show that at $167 \mathrm{~km}$, the $\mathrm{N}$ and $\mathrm{O}$ densities reach a minimum near 2 a.m., rather than a maximum as the above simple interpretation suggests. However, the density scale heights also reach a minimum near midnight, and if the ONMS measurements are extrapolated to $140 \mathrm{~km}$ (the upper boundary of our model), we find that both the $\mathrm{N}$ and $\mathrm{O}$ densities rise from dusk to maxima near 10 p.m. and decline toward dawn; the

TABLE 1. Table of Chemical Reactions

\begin{tabular}{lll}
\hline \multicolumn{1}{c}{ Reaction } & \multicolumn{1}{c}{ Rate Coefficient } & \multicolumn{1}{c}{ Source } \\
\hline (1) $\mathrm{O}+\mathrm{N} \rightarrow \mathrm{NO}$ & $2.5 \times 10^{-17} \mathrm{~cm}^{3} \mathrm{~s}^{-1}$ & Mandelman et al. [1973] \\
$(2) \mathrm{N}+\mathrm{NO} \rightarrow \mathrm{N}_{2}+\mathrm{O}$ & $2.1 \times 10^{-11} \mathrm{~cm}^{3} \mathrm{~s}^{-1}$ & Phillips and Schiff [1962] \\
(3) $\mathrm{O}+\mathrm{N}+\mathrm{CO}_{2} \rightarrow \mathrm{NO}+\mathrm{CO}_{2}$ & $1.1 \times 10^{-32}(T / 300)^{-1 / 2} \mathrm{~cm}^{6} \mathrm{~s}^{-1}$ & Baulch et al. [1973] \\
(4) $\mathrm{O}+\mathrm{O}+\mathrm{CO}_{2} \rightarrow \mathrm{O}_{2}+\mathrm{CO}_{2}$ & $4.7 \times 10^{-33}(T / 300)^{-2} \mathrm{~cm}^{6} \mathrm{~s}^{-1}$ & Campbell and Gray [1973] \\
(5) $\mathrm{O}+\mathrm{CO}+\mathrm{CO}_{2} \rightarrow \mathrm{CO}_{2}+\mathrm{CO}_{2}$ & $2 \times 10^{-37} \mathrm{~cm}^{6} \mathrm{~s}^{-1}$ & Simonaites and Heicklen [1972] \\
(6) $\mathrm{O}+\mathrm{O}_{2}+\mathrm{CO}_{2} \rightarrow \mathrm{O}_{3}+\mathrm{CO}_{2}$ & $1.4 \times 10^{-33}\left(T_{N} / 300\right)^{-25} \mathrm{~cm}^{6} \mathrm{~s}^{-1}$ & Kaufman [1969] \\
(7) $\mathrm{O}+\mathrm{O}_{3} \rightarrow \mathrm{O}_{2}+\mathrm{O}_{2}$ & $1.9 \times 10^{-11} \exp (-2300 / T) \mathrm{cm}^{3} \mathrm{~s}^{-1}$ & Hampson [1973] \\
\hline
\end{tabular}




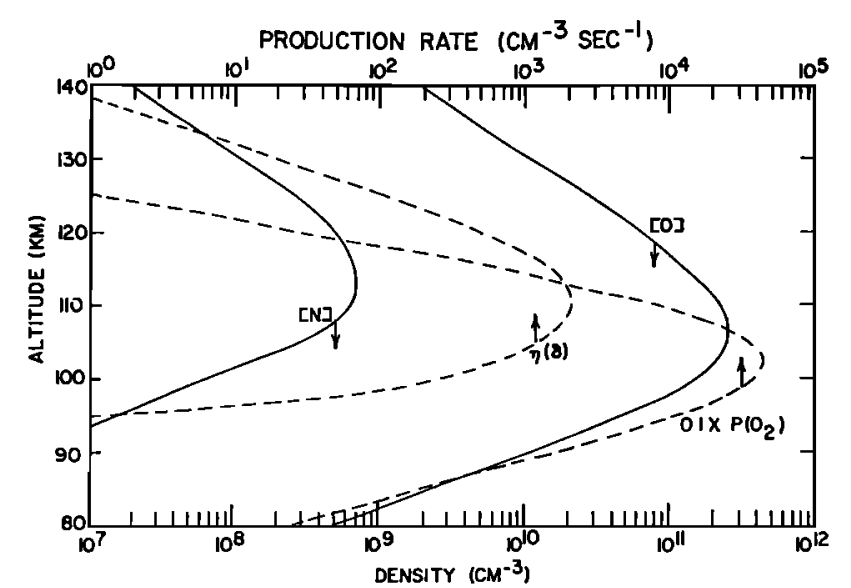

Fig. 3. Calculated profiles of the densities of atomic oxygen and atomic nitrogen. The volume emission rate of the NO $\delta$ bands is due to reaction 1 , and the production of $\mathrm{O}_{2}$ molecules is due to reaction 4 for an eddy diffusion coefficient of $3 \times 10^{8} \mathrm{~cm}^{2} \mathrm{~s}^{-1}$ at $140 \mathrm{~km}$.

maxima are quite pronounced in that the 10 p.m. densities $\left(\sim 1 \times 10^{8} \mathrm{~cm}^{-3}\right.$ and $\sim 1 \times 10^{10} \mathrm{~cm}^{-3}$ for $\mathrm{N}$ and $\left.\mathrm{O}\right)$ exceed those at 8 p.m. and at 2 a.m. by a factor of about 2 . From this we would anticipate the peak in the airglow emission to occur before midnight rather than after.

Second, the Herzberg II emission from molecular oxygen observed from Venera 9 [Krasnopolsky et al., 1976] shows a peak near 11 p.m. and a steady decline from that time until 4 a.m. This is consistent with the extrapolated ONMS measurements of $O$ but not with the average NO brightness map. We may note, however, that we occasionally see NO airglow brightness distributions similar to the Venera distribution; on orbit 184 , for example, the bright spot lies at or slightly before midnight. It would be of great interest to know what the average distribution was for the several dawn-to-dusk passes made by Venera 9 . The average intensity of $\sim 5 \mathrm{kR}$ seen by Venera 9 represents a quantum efficiency of $\sim 10^{-2}$ photons/O atom, given our $O$ flux of $1 \times 10^{12} \mathrm{~cm}^{-2} \mathrm{~s}^{-1}$. A laboratory determination (G. M. Lawrence, private communication, 1979) suggests

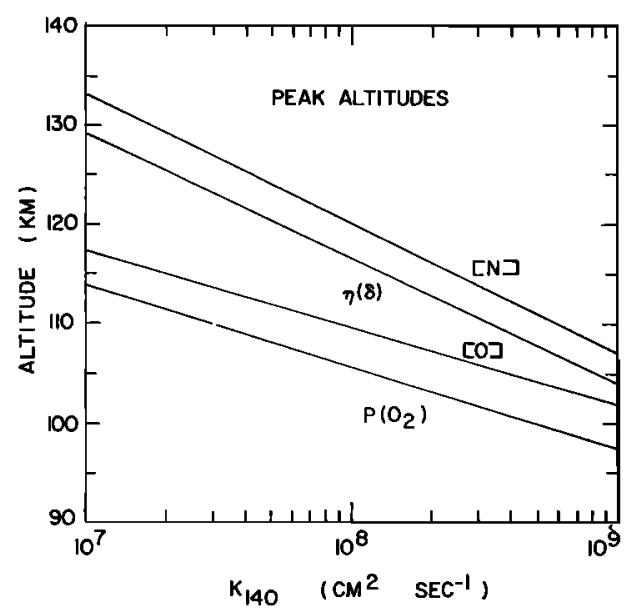

Fig. 4. The dependence of the altitudes of the maximum densities of oxygen and nitrogen, the maximum $\delta$ band emission rate, and the maximum $\mathrm{O}_{2}$ production rate as a function of the eddy diffusion coefficient.
ALTITUDE OF EMITTING LAYER

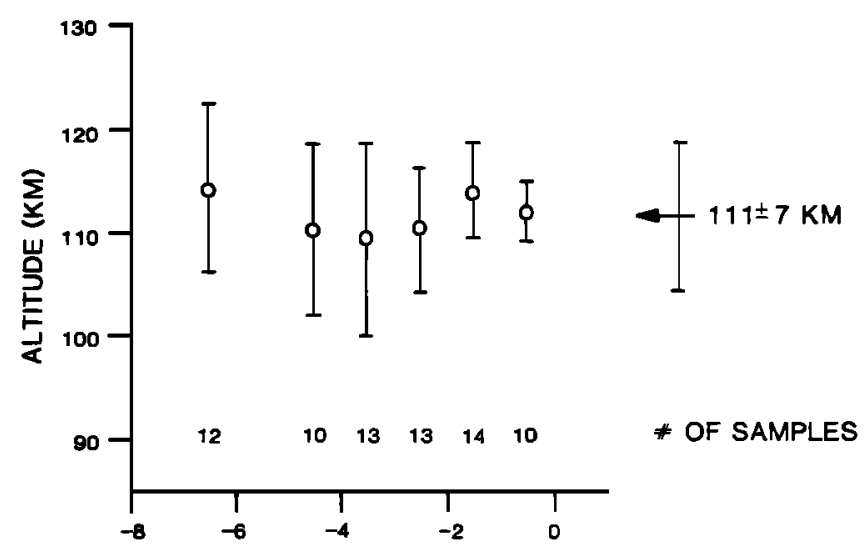

TME FROM PERIAPSIS (MIN)

Fig. 5. Measurements of the altitude of the emitting layer, grouped in 1-min bins. At $P-6.5 \mathrm{~min}$, the limbs are at $35^{\circ} \mathrm{N}$; at $P-$ $.5 \mathrm{~min}$, they are at $12^{\circ} \mathrm{N}$.

a value an order of magnitude smaller than this, but the uncertainty is large.

A third relevant observation is the detection of the $1.27 \mu$ emission from molecular oxygen on the nightside of Venus by Connes et al. [1979]. Their field of view lay in the postmidnight sector and the mean zenith intensity was $\sim 600 \mathrm{kR}$. If we assume a unit efficiency for the production of $1.27 \mu$ photons upon recombination of molecular oxygen, then the necessary flux of $O$ is $1.2 \times 10^{12} \mathrm{~cm}^{-2} \mathrm{~s}^{-1}$, entirely compatible with our model and, by implication, with the ONMS measurements also. Thus given the simplicity of our model and the possibility of other sources for the $\mathrm{O}_{2}$ emissions [Connes et al., 1979] there is a fair degree of consistency between the measured $\mathrm{O}$ and $\mathrm{N}$ densities and the measured $\mathrm{NO}$ and $\mathrm{O}_{2}$ airglow intensities.

The discrepancies between the local time dependencies of the $\mathrm{NO}$ and $\mathrm{O}_{2}$ emissions and the ONMS measurements are difficult to assess in the absence of an adequate dynamical model. The descending part of the thermospheric circulation might be displaced toward the morning terminator if the 4day retrograde rotation seen at the cloud top $(\sim 65 \mathrm{~km})$ level persists to higher altitudes. However, our current ideas cannot account for morphological differences between the NO and $\mathrm{O}_{2}$ emissions, and we are forced to invoke the day-to-day variability of the morphology to explain the observed discrepancy.

The variations in morphology and overall brightness are themselves of interest. Our simple model shows that the brightness of the NO emission depends only on the downward flux of $\mathrm{N}$ atoms; thus the observed patchiness of the emission must result from spatial irregularities in the high-altitude circulation pattern. This interpretation is consistent with the variability of the nightside $\mathbf{N}$ and $\mathbf{O}$ concentrations observed by the ONMS. By the same token, the variations in overall brightness imply variations in the supply of $\mathrm{N}$ atoms from the dayside, perhaps indicative of variations in the relative importance of global and local transport processes in the dayside thermosphere. Such variations would certainly affect the global transport of $N$ (and $O$ ), since the dissociation of $\mathbf{N}_{2}$ 
peaks at altitudes near the location of the homopause inferred by von Zahn et al. [1979].

Acknowledgments. This research was performed in part under NASA contract NAS2-9477 and supported in part by NASA grant NGL 06-003-052. The National Center for Atmospheric Research (NCAR) is acknowledged for providing computing services. NCAR is sponsored by the National Science Foundation. J.-C. G. is supported by the Belgian Foundation for Scientific Research (FNRS).

The Editor thanks D. E. Anderson and P. D. Feldman for their assistance in evaluating this paper.

\section{REFERENCES}

Barth, C. A., J. B. Pearce, K. K. Kelly, L. Wallace, and W. G. Fastie, Ultraviolet emissions observed near Venus from Mariner V, Science, 158, 1675, 1968.

Baulch, D. L., D. D. Drysdale, D. G. Horne, and A. C. Lloyd, Homogenous gas phase reactions of the $\mathrm{H}_{2}-\mathrm{N}_{2}-\mathrm{O}_{2}$ system, in Evaluated Kinetic Data for High Temperature Reactions, vol. 2, pp. 1557, Chemical Rubber Company Press, Cleveland, Ohio, 1973.

Campbell, I. M., and C. N. Gray, Rate constants for $O\left({ }^{3} P\right)$ recombination and association with $\mathrm{N}\left({ }^{4} S\right)$, Chem. Phys. Lett., 18, 607, 1973.

Colin, L., Encounter with Venus, Science, 203, 743, 1979a.

Colin, L., Encounter with Venus: An update, Science, 205, 44, $1979 b$.

Connes, P., J. F. Noxon, W. A. Traub, and N. P. Carleton, $\mathbf{O}_{2}\left({ }^{\prime} \Delta\right)$ emission in the day and night airglow of Venus, Astrophys. J. Lett., in press, 1980.

Dickinson, R. G., and E. C. Ridley, Venus mesosphere and mesosphere temperature structure, 2, Day-night variations, Icarus, 30, 163-178, 1977.

Feldman, P. D., H. W. Moos, J. T. Clarke, and A. L. Lane, Identification of the UV nightglow from Venus, Nature, 279, 221, 1979.

Hampson, R. F. (Ed.), Survey of photochemical and rate data for twenty eight reactions of interest in atmospheric chemistry, $J$. Phys. Chem. Ref. Data, 2, 267-312, 1973.

Kaufman, F., Elementary gas reactions, Ann. Rev. Phys. Chem, 20, 45-90, 1969.

Krasnopolsky, V. A., A. A. Krysko, V. N. Rogachev, and V. A. Parshev, Observations of Venus from the interplanetary spacecraft Venera 9 and Venera 10, Sov. Space Res., 14, 789, 1976.
Lawrence, G. M., C. A. Barth, and V. Argabright, Excitation of the Venus night airglow, Science, 195, 573, 1977.

Mandelman, M., T. Carrington, and R. A. Young, Predissociation and its inverse, using resonance absorption $\mathrm{NO}\left(\mathrm{C}^{2} \Pi\right) \rightleftarrows \mathrm{N}+\mathrm{O}, J$. Chem. Phys., 58, 84, 1973.

Niemann, H. B., W. T. Kasprzak, A. E. Hedin, D. M. Hunten, and N. W. Spencer, Mass spectrometric measurements of the neutral gas composition of the thermosphere and exosphere of Venus, $J$. Geophys. Res., this issue.

Phillips, L. F., and H. I. Schiff, Mass spectrometer studies of atom reactions, 1, Reactions in the atomic nitrogen-ozone system, $J$. Chem. Phys., 36, 1509, 1962.

Rishbeth, H., and D. W. Barron, Equilibrium electron distributions in the ionospheric F2-layer, J. Atmos. Terr. Phys., 18, 234, 1960.

Rusch, D. W., and T. E. Cravens, A model of the neutral and ion nitrogen chemistry in the daytime thermosphere of Venus, Geophys. Res. Lett., 6, 791, 1979.

Simonaites, R., and J. Heicklen, Kinetics and mechanisms of the reaction of $\mathrm{O}\left({ }^{3} P\right)$ with carbon monoxide, $J$. Chem. Phys., 56, 2004-2011, 1972.

Slanger, T. G., and G. Black, The $\mathrm{O}_{2}\left(C^{3} \Delta_{u} \rightarrow a^{\prime} \Delta_{g}\right)$ bands in the nightglow spectrum of Venus, Geophys. Res. Lett., 5, 947, 1978.

Stewart, A. I. F., Design and operation of the Pioneer Venus Orbiter Ultraviolet Spectrometer, IEEE Trans. Geosci. Remote Sensing, $G E-18,65,1980$

Stewart, A. I., and C. A. Barth, Ultraviolet night airglow of Venus, Science, 205, 59, 1979.

Stewart, A. I., D. E. Anderson, Jr., L. W. Esposito, and C. A. Barth, Ultraviolet spectroscopy of Venus: Initial results from the Pioneer Venus orbiter, Science, 203, 777, 1979.

von Zahn, V., K. H. Fricke, H.-J. Hofimann, and K. Pelka, Venus: Eddy coefficients in the thermosphere and the inferred helium content of the lower atmosphere, Geophys. Res. Lett., 6, 337-340, 1979.

von Zahn, V., K. H. Fricke, D. M. Hunten, D. Krankowsky, K. Mauersberger, and A. O. Nier, The upper atmosphere of Venus during morning conditions, $J$. Geophys. Res., this issue.

(Received January 2, 1980;

revised May 28, 1980;

accepted May 29, 1980.) 